\title{
Some aspects of mass-energy equivalence which appears in left-handed metamaterials
}

\author{
Naghi Gasimov ${ }^{1, *}$, Muharrem Karaaslan ${ }^{2}$, Cumali Sabah $^{3}$, and Faruk Karadag ${ }^{1}$ \\ ${ }^{1}$ Department of Physics, Çukurova University, Saricam, Adana 01330, Turkey \\ 2 Department of Electrical and Electronics Engineering, Iskenderun Technical University, Iskenderun, Hatay 31200, Turkey \\ ${ }^{3}$ Department of Electrical and Electronics Engineering, Middle East Technical University-Northern Cyprus Campus \\ (METU-NCC), Kalkanli, Guzelyurt 99738, TRNC/Mersin 10, Turkey
}

Received: 1 December 2017 / Accepted: 5 April 2019

\begin{abstract}
In this work, the concept of mass-energy equivalence in left-handed metamaterials is discussed by following Einstein's box thought experiment. Left-handed metamaterials are artificial composite structures that exhibit unusual properties, especially negative refractive index, in which phase and group velocities are directed oppositely. Equation $E=m c^{2}$ assumes that, in vacuum, the propagation of an electromagnetic radiation from emitter to receiver is accompanied by the transfer of mass. It was hypothesized previously that if the space between emitter and receiver is medium with a negative refractive index, then radiation transfers the mass not from the emitter to receiver as expected, but rather from the receiver to the emitter due to the opposite directions of phase and group velocities. In this paper, it is shown that even though one radiating atom is taken, the negative mass transferring must be in force. In particular, it means that, if the atom radiates a photon in a medium with negative refractive index, photon transfers the mass not from the atom, but to the atom.
\end{abstract}

Keywords: Mass-energy equivalence / metamaterials / negative refractive index / special theory of relativity

\section{Introduction}

In 1905 , the theory of special relativity was postulated [1] by Albert Einstein. In the same year another paper was published by Einstein that contained the mass-energy equivalence equation [2]

$$
E=m c^{2}
$$

which was the direct result of special relativity. In 1906, Einstein proposed a thought experiment [3], known as the "Einstein's box" (or photon in a box), which also led to equation (1). Equation (1), in particular, assumes that if the space between the emitter and receiver is a vacuum, then the propagation of electromagnetic radiation with energy $E$ from emitter to receiver is accompanied by the transfer of a mass equal to $m=E / c^{2}$.

Einstein's box thought experiment was considered in a vacuum, where the phase and group velocities of radiation were equal to $v_{\mathrm{ph}}=v_{\mathrm{gr}}=c$ (speed of light in a vacuum). The question is the following: what will change if we repeat this

\footnotetext{
* e-mail: ngasimov@student.cu.edu.tr
}

thought experiment in a medium with refractive index $n$ where the phase and group velocities of the radiation differ from the speed of light in a vacuum?

In 1967, Veselago theoretically described the main properties of materials with negative refractive index [4]. In 2000 , the possibility of negative refraction was confirmed experimentally by Smith Willie et al. [5]. These materials are also called as left-handed metamaterials. Left-handed metamaterials are artificial composite structures that exhibit opposition of phase and group velocities of radiation. The obvious question now is: what will be changed in the result of Einstein's box thought experiment, if the medium is made of metamaterial not with positive refractive index, but with negative refractive index $n$, where the phase and group velocities are in opposite direction?

These problems are related to the problem of transferring energy, linear momentum, and mass as the photon propagates in medium, which is discussed in [6-8], and some rather strong statements are obtained. In particular, it was obtained that equation (1) should be changed with

$$
E=m v_{\mathrm{ph}} v_{\mathrm{gr}}
$$




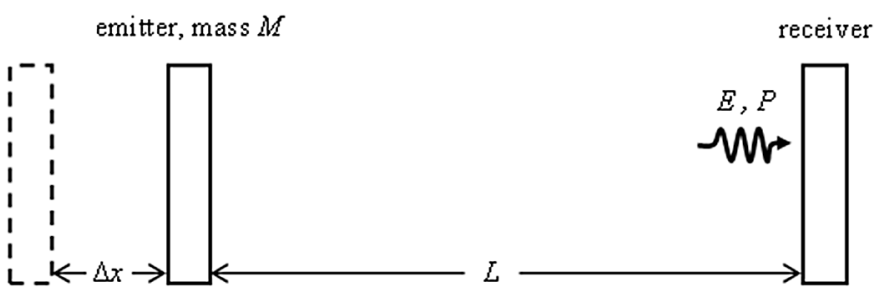

Fig. 1. Photon with energy $E$, linear momentum $P$, propagates from emitter to receiver and emitter recoils to the left.

in common case and, moreover, due to opposite directions of phase $v_{\mathrm{ph}}$ and group $v_{\mathrm{gr}}$ velocities in medium with negative refractive index, radiation transfers mass not from the emitter to the receiver as usually, but from the receiver to the emitter.

In this work, it is shown that if the previous statements are correct, then these results must be applicable also for simple "radiating atom". In other words, existing of receiver in this thought experiment is not necessary and even by "forgetting" the receiver and "decreasing" the emitter to one atom, all the previous statements must be in force. In particular, it means that if the atom radiates photon inside negatively refracting medium, because of the opposition of phase and group velocities, photon transfers the mass not from an atom, but to an atom. In other words, the mass of the atom increases with emission of a photon and decreases with the absorption of a photon.

\section{Einstein's box thought experiment in vacuum: derivation of $E=m c^{2}$}

Since 1906, several modifications of Einstein's box thought experiment have been proposed. At first, we will repeat derivation of mass-energy equivalence equation in vacuum following to [3] and using French's "unhinged box" modification [9].

Suppose that photon with energy $E$ and linear momentum

$$
P=\frac{E}{c}
$$

have been emitted from emitter with mass $M$ (Fig. 1). As a result the emitter receives a velocity

$$
V=\frac{P}{M}=\frac{1}{M} \frac{E}{c}
$$

in opposite direction of the photon and shifts to the left. The distance between emitter and receiver is $L$ and obviously photon will reach the receiver in time

$$
t=\frac{L}{c}
$$

During this time $t$, emitter will shift to the left such distance

$$
\Delta x=V t \text {. }
$$

If we substitute velocity (4) and time (5) in (6) then we obtain

$$
\Delta x=\frac{E L}{M c^{2}} .
$$

This equation can be rewritten as

$$
\Delta x M=\frac{E}{c^{2}} L
$$

According to the center of mass theorem, if we assume that during this process the center of mass of a whole system (emitter, receiver, and photon) will not be changed, it is necessary to imply that the mass

$$
m=\frac{E}{c^{2}}
$$

should be to move a distance $L$ to the right from emitter to receiver. In other words, emitter lost the mass $m$, while receiver acquires the mass $m$ during propagation energy $E$ from emitter to receiver.

\section{Einstein's box thought experiment in medium}

In equation (9) there are two $c$ 's. One of the $c$ s comes from equation (3), which is phase velocity $v_{\mathrm{ph}}$, and the other one comes from equation (5), which is group velocity $v_{\mathrm{gr}}$. It is clear that, when light propagates in vacuum, both velocities are equal to the speed of light in vacuum $v_{\mathrm{ph}}=v_{\mathrm{gr}}=c$. But if the space between emitter and receiver filled with medium, then phase and group velocities of light differ from the speed of light in vacuum $c$. So, we should change one of the c's with $v_{\mathrm{ph}}$ and the other one with $v_{\mathrm{gr}}$. In result we get the following equation

$$
m=\frac{E}{v_{\mathrm{ph}} v_{\mathrm{gr}}}
$$

for the transferring mass [6-8]. Therefore, it turns out that equation (1) is a special case of common equation (10).

As it was said before, direction of phase and group velocities is antiparallel in mediums with negative refractive index (or left-handed metamaterials). Thus, if the refractive index of medium between emitter and receiver is negative, then transferred mass $m$ also is negative. This situation leads to the strange result: if the emitter radiates in medium with negative refractive index, instead of losing mass, it acquires mass.

These problems closely related to the concept of photon linear momentum in medium, wave-particle duality, and Abraham-Minkowski controversy which is discussed in the next sections.

\section{Phase and group velocity inside waveguide and plasma}

It is well known that the phase and group velocity of electromagnetic waves inside an empty waveguide is 
determined with the following equations [10]

$$
\begin{gathered}
v_{\mathrm{ph}}=\frac{c}{\sqrt{1-\omega_{c}^{2} / \omega^{2}}} \\
v_{\mathrm{gr}}=c \sqrt{1-\omega_{c}^{2} / \omega^{2}}
\end{gathered}
$$

where $c$ is the speed of light in vacuum, $\omega_{\mathrm{c}}$ is the cutoff frequency, below which electromagnetic waves cannot propagate through waveguide, and $\omega$ is the frequency of propagating waves. It is seen from equations (11) and (12) that [10]

$$
v_{\mathrm{ph}} v_{\mathrm{gr}}=c^{2}
$$

which satisfies equation (10). The similar equations are valid for phase and group velocities in a plasma [11]. Therefore, we can be sure that equation (10) is correct at least for an empty waveguides and plasmas. These facts can be considered with caution as the support of equation (10).

\section{Momentum of a photon in a medium: $p=n E / c$ or $p=E / n c$ ?}

There is a debate about linear momentum of a photon in a medium which continues till today [12-14]. These discussions, known as the Abraham-Minkowski controversy, lead to the following equations:

$$
\begin{gathered}
\text { Minkowski : } p=\frac{n E}{c} \\
\text { Abraham: } p=\frac{E}{n c}
\end{gathered}
$$

where $n$ is refractive index of medium. Equation (10) was obtained by using Minkowski form (14). The question is: which is correct, equation (14) or equation (15)? Moreover, what will be changed in our result if we use the Abraham form, i.e., equation (15)? We can forget for a moment about this controversy and consider our attention to the following two theories: wave-particle duality theory and quantum theory of Cherenkov radiation.

First of all, it should be noted that without considering Abraham-Minkowski controversy, we must take into account that equation (14) is the direct result of the wave-particle duality theory [15]. In fact

$$
\begin{gathered}
p=\hbar k \\
E=\hbar \omega .
\end{gathered}
$$

From equations (16) and (17), we get

$$
p=\frac{k E}{\omega} .
$$

It is clear that $\omega / k$ is the phase velocity. So equation (18) can be written as

$$
p=\frac{E}{v_{\mathrm{ph}}}=\frac{n E}{c}
$$

which is the same with equation (14). Therefore, waveparticle duality theory implies that the momentum of a photon in a medium should be determined as equation (14) which is corresponds to Minkowski form. Since the wave-particle duality together with vacuum covers also mediums, this theory can be considered as a support for equation (14).

The second theory which is worthy of attention is the quantum theory of Cherenkov radiation which was proposed by Ginzburg in 1940 [16]. It is important to note that the quantum theory of Cherenkov radiation can be proposed only with accepting that the momentum of a photon in a medium is determined with

$$
p=\hbar k=\frac{n \hbar \omega}{c}
$$

which is corresponds to equation (14). The quantum theory of Cherenkov radiation is the well-known and wellestablished theory and therefore it can be regarded as a strong support of equation (14).

Thus, without entering to Abraham-Minkowski controversy discussions we can see some "glimpse" that the Minkowski form is correct. For additional information about Abraham-Minkowski controversy and tensor calculations one can refer to $[6,7,14,17]$.

After all, it is important to note that if we accept that the linear momentum of a photon in a medium is determined not with equation (14), but with equation (15), even in this case mass transferring is also taken place but the value of transferring mass will be different. It is not difficult to show with analogous approach that if we use equation (15), then the value of transferring mass will be determined as

$$
m=\frac{v_{\mathrm{ph}} E}{v_{\mathrm{gr}} c^{2}} .
$$

Therefore, mass transferring phenomenon will take place even if it is assumed that the correct one is equation (15). We can calculate the difference of transferring mass value between two approaches by dividing equations (10) and (21) with each other

$$
\frac{E}{v_{\mathrm{ph}} v_{\mathrm{gr}}} \div \frac{v_{\mathrm{ph}} E}{v_{\mathrm{gr}} c^{2}}=\frac{c^{2}}{v_{\mathrm{ph}}^{2}}=n^{2} .
$$

Thus, transferring mass will be $n^{2}$ times less in Abraham form, wherein the direction of mass transferring is still be negative.

\section{Derivation of $E=m c^{2}$ with radiating atom configuration}

Position of the center of mass of whole system should be stationary not only before emission of photon from emitter 


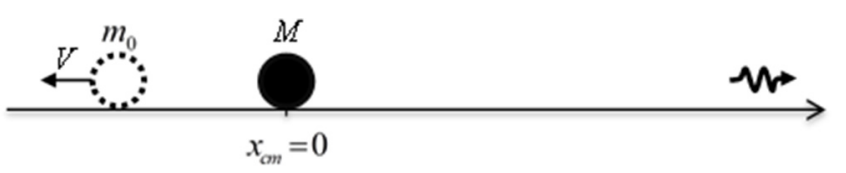

Fig. 2. The atom emits a photon reducing the mass and recoils to the left.

and after absorption in receiver, but also during propagation of a photon between emitter and receiver. It means that actually the receiver in French's modification is "redundant". Thus, by discarding receiver and assuming the emitter as an atom, it is possible to simplify Einstein's box thought experiment till one atom [18]. In result we get one radiating atom and, in this configuration, the mass transferring gets relatively a clear physical picture.

Suppose the resting atom of mass $M$ located on origin of one-dimensional coordinate system (Fig. 2). Obviously, the position of center of mass should be at $x_{\mathrm{cm}}=0$. Atom radiates a photon of energy $E$ at time $t=0$. After emission of photon, the mass of atom decreased to $m_{0}$ and it recoils with a velocity (assuming that the velocity of the atom is very small, $V \ll c)$

$$
V=-\frac{P}{m_{0}}=-\frac{E}{m_{0} c}
$$

The position of center of mass $x_{\mathrm{cm}}^{\prime}$ after emission is given by (for atom-photon system)

$$
m_{0} V t+m c t=M x_{\mathrm{cm}}^{\prime}
$$

The theorem of the center of mass requires that $x_{\mathrm{cm}}^{\prime}=x_{\mathrm{cm}}$. But $x_{\mathrm{cm}}=0$, thus

$$
m_{0} V t+m c t=0
$$

If we substitute equation (23) in equation (25) then we obtain for transferring mass

$$
m=\frac{E}{c^{2}}
$$

This result leads us to an assumption which is discussed in the next section.

\section{Radiating atom inside left-handed metamaterial}

Referring to previous results, one can come to the interesting assumption: if the atom emits photon inside medium with a negative refractive index, this atom emits photon by acquiring mass. The reverse must also be valid: if the atom absorbs the photon, then atom will decrease its mass. It should be noted that, during mass transferring, the photon itself is massless [19].

It is interesting to note that each unit cell of threedimensional metamaterial, in fact, can be considered as an "atom" of metamaterial, so every internal "atom" of lefthanded metamaterial actually located inside own medium of negative refraction. It turns out that the light source, made of the left-handed metamaterial, should acquire mass during radiation. It is possible at all that there are exotic applications which lie behind the negative mass transmission phenomenon.

\section{Can the emitter move freely inside the left-handed medium?}

In previous sections, we discussed Einstein's box thought experiment in left-handed medium implying that the emitter can move in this medium freely. However, the left-handed metamaterials which are known today are solid. Therefore, an important and interesting question arises: how is it possible that the emitter can freely move backward without sharing the momentum with the surrounding medium? In our opinion this situation can be clarified by at least three approaches.

First of all, it must be noted that despite the left-handed metamaterials are solid, they are not completely filled. There is certain symmetric empty volumes inside lefthanded metamaterials (usually empty volumes bigger than "meta-atoms" in terahertz metamaterials) [5] and since the size of the emitter can be smaller than the characteristic lattice parameter of metamaterial, in principle it is possible to locate the emitter to the empty part of left-handed medium. In this case the emitted photon will "feel" itself in negative refractive index medium, while emitter will be able to move backward freely.

The second approach is related to fluids. It is clear that the emitter can be able to move through the liquid, but with fluid resistance. However, we can avoid this resistance. It is known from low temperature physics [20] that helium (and some other Bose-Einstein condensates) below certain temperature becomes to a new state which is called superfluid (or quantum fluid), whose viscosity is zero. This means that a solid body that moves through that superfluid (below certain velocity) will not experience fluid resistance. Therefore, no momentum sharing will be with surrounding medium, that is, the solid body (or emitter) will move as in a vacuum [21]. Of course, for now we do not have liquid metamaterials, not to mention liquid metamaterials with a negative refractive index and perhaps last considerations are very premature, but it is not excluded that we will have such mediums in future.

Finally, it should be added that it is not necessary that the emitter completely immersed into the left-handed medium. It is enough that the emitter is located at the boundary vacuum-left-handed medium, and the radiation occurred inside the left-handed medium as described in [6] which corresponds to French's configuration [9]. In this case radiated photon will propagate in the left-handed medium, while the emitter can be able to move backward freely in vacuum.

\section{Analysis}

One can see that one of the key points in Einstein's box thought experiment is the motionless of the position of center of mass. As stated in the beginning, mass-energy 
equivalence equation is the direct result of special relativity. But, it is interesting to recall that the postulates of the special relativity theory were not used here for derivation of equation (26). This situation was noted in [7], and also in [22]. But how does the classical and relativistic approach lead to the same equation?

In our opinion, this is because that the key point here is the application of classical center of mass theorem to photon, which is massless. In this point it is appropriate to recall that the postulates of special relativity theory follow from the classical Galilean principle of relativity which is generalized for electromagnetic processes by Einstein [23]. Here is the similar situation with Einstein's box thought experiment: we apply the classical center of mass theorem (which is applicable only to classical bodies having mass) to atom-photon system, where the photon is massless, but at the same time has a linear momentum. Thus, we apply classical theory to nonclassical particle (photon) which cannot be considered as "classical" operation. Obviously, if we require that the center of mass does not change, we automatically need to assign a mass to the photon, and this approach leads to the mass-energy equivalence equation.

The authors would like to express their gratitude to Prof. V.G. Veselago for useful discussions and advice. The authors would also like to acknowledge the Scientific and Technological Research Council of Turkey (TÜBİTAK) and the Scientific Project Unit of Çukurova University (FDK-2017-8948) for full financial support.

\section{References}

1. A. Einstein, Ann. Phys. 17, 891 (1905)
2. A. Einstein, Ann. Phys. 18, 639 (1905)

3. A. Einstein, Ann. Phys. 20, 627 (1906)

4. V.G. Veselago, Sov. Phys. Usp. 10, 509 (1968)

5. D.R. Smith Willie, J. Padilla, D.C. Vier, S.C. Nemat-Nasser, S. Schultz, Phys. Rev. Lett. 84, 4184 (2000)

6. V.G. Veselago, Phys. Usp. 52, 649 (2009)

7. V.G. Veselago, Phys. Usp. 54, 1161 (2011)

8. V.G. Veselago, EPJ Appl. Metamat. 1, 9 (2014)

9. A.P. French, Special relativity, The M.I.T. Introductory Physics Series (W. W. Norton \& Company, Inc., New York, 1968), pp. $27-28$

10. S.J. Orfanidis, Electromagnetic waves and antennas (Rutgers University, New Brunswick, NJ, 2016), pp. 379381

11. R. Fitzpatrick, Plasma physics: an introduction (CRC Press, Boca Raton, FL, 2015), pp. 154-157

12. U. Leonhardt, Nature 444, 823 (2006)

13. B.A. Kemp, Nat. Photon. 10, 291 (2016)

14. M.G. Silveirinha, Phys. Rev. A 96, 033831 (2017)

15. R.P. Feynman, The Feynman lectures on physics (AddisonWesley, Menlo Park, CA, 1977), pp. 34-11

16. V.L. Ginzburg, Sov. Phys. Usp. 2, 874 (1960)

17. V.G. Veselago, V.V. Shchavlev, Phys. Usp. 53, 317 (2010)

18. A.F. Antippa, Am. J. Phys. 44, 841 (1976)

19. L.B. Okun, Phys. Usp. 43, 1270 (2000)

20. L.D. Landau, E.M. Lifshitz, Fluid mechanics, Course of Theoretical Physics, Vol. 6 (Pergamon Press, Oxford, 1987), pp. $515-517$

21. D. Ceperley, Phys. World 11, 19 (1998)

22. L.I. Mandelstam, Lectures on optics, relativity theory and quantum mechanics (Nauka, Moscow, 1972), pp. 144-145 (in Russian)

23. V.A. Ugarov, Special theory of relativity (Mir Publishers, Moscow, 1979), pp. 29-32

Cite this article as: Naghi Gasimov, Muharrem Karaaslan, Cumali Sabah, Faruk Karadag, Some aspects of mass-energy equivalence which appears in left-handed metamaterials, EPJ Appl. Metamat. 6, 16 (2019) 